\title{
Loneliness and social integration as mediators between physical pain and suicidal ideation among elderly men
}

\author{
Mira Lutzman, ${ }^{1,2}$ Eliane Sommerfeld, ${ }^{1}$ (i) and Sarah Ben-David ${ }^{3}$ \\ ${ }^{1}$ Department of Psychology, Ariel University, Ariel, Israel \\ ${ }^{2}$ Sheba Medical Center, Tel Hashomer, Israel \\ ${ }^{3}$ Department of Criminology, Ariel University, Ariel, Israel
}

Abstract

Objectives: Suicide in the elderly is a complex and significant public health problem. The purpose of our study was to examine the role of loneliness and social integration as potential mediators in the relationship between physical pain and suicidal ideation in the elderly.

Design: Descriptive, bivariate correlations, and moderated mediation analyses were performed.

Setting: Personal meetings were held with participants in their homes.

Participants: A total of 198 elderly men aged 65 and over.

Measurements: Self-report measures: Beck Scale for Suicidal Ideation, Physical pain subscale, Multidimensional Social Integration in Later Life Scale, and University of California, Los Angeles (UCLA) Loneliness Scale (Version 3).

Results: Our findings showed that the association between physical pain and suicidal ideation was mediated by loneliness and social integration. Further analyses revealed that this mediation model was significant among single, but not married, men.

Conclusions: Physical pain and social factors are both important in understanding suicidality in late life. Elderly single men who experience physical pain may be lonelier and less socially integrated, and these factors may contribute to higher risk of suicidal ideation.

Key words: physical pain, suicidal ideation, loneliness, social integration

\section{Introduction}

Elderly men aged 65 and over exhibit the highest suicide rates of all ages (WHO, 2014), with the suicide risk of older men being four times higher than women (Law et al., 2016). Although a serious global issue, suicide in old age is a rather neglected area. Understanding of suicidality requires an indepth examination of multiple risk factors. Since both physical and psychosocial factors are known to contribute to suicidality in general (Bahk et al., 2011; Lewitzka et al., 2017), our goal in the present study was to examine how loneliness and social integration may act as potential mediators in the association between physical pain and suicidality in elderly men.

Correspondence should be addressed to: Eliane Sommerfeld, Ariel University, Ramat, Hagolan 65, Ariel, Israel. Email: sommerfelde@ariel.ac.il. Received 16 Feb 2020; revision requested 12 Apr 2020; revised version received 03 Jun 2020; accepted 05 Jun 2020. First published online 09 Jul 2020
Physical pain among elderly people leads to deterioration in functioning, mobility, and quality of life, as well as to sadness and loneliness (Rapo-Pylkk et al., 2016). Moreover, dealing with diseases and chronic pain is a well-known risk factor for depression and suicide in the elderly (Erlangsen et al., 2015; Chan et al., 2011; Lee and Moon, 2015). For example, elderly who attempted suicide reported poorer health in terms of vascular and respiratory diseases (Bergman Levy et al., 2011). Waern and colleagues (2002) pointed out that visual impairment, neurological disorders, and malignant disease were independently associated with increased risk of suicide in the elderly. Specific consequences of physical illness might also contribute to suicide risk. In particular, physical pain is a significant risk factor for suicidality among the elderly (Racine, 2018). According to Kim (2016), extreme pain as well as moderate limitation in usual activities increased the risk of suicidal ideation and suicide attempts in the elderly, over and above the existence of chronic diseases and 
depression. Even so, it is still unclear what processes might explain the association between physical pain and suicidality.

A possible explanation of the association between physical pain and suicidality among the elderly might be that physical pain limits individual involvement in social activities and relationships. The investigation of social risk and protective factors for suicide can be accessed from an objective (e.g. frequency of social interactions) as well as subjective perspective (e.g. feelings of loneliness), which may also be connected. Elderly people are forced to deal with a variety of losses, including the loss of close relationships or death of a partner, which often lead to the experience of loneliness. In late life, participating in social and leisure activities, hobbies, and frequency of interpersonal interactions beyond the family indicate well-being (Zhang and Zhang, 2015), although these might be limited due to physical pain. Moreover, previous research has shown that subjective perception of health and number of chronic illnesses were also found as predictors of loneliness in old age (Theeke, 2009; Emerson et al., 2018). Elderly people with chronic pain reported poor mobility and felt sadder, lonelier, and more tired (Rapo-Pylkk et al., 2016). According to Domènech-Abella and colleagues (2019), objective and perceived social isolation was associated with affective disorders, specifically with anxiety and depression among the elderly. Reduced social connectedness was associated with suicidal ideation, non-fatal suicidal behavior, and suicide in later life (Dennis et al., 2005). Subjective feelings of loneliness were also found to be a significant risk factor for suicide in the general adult population (Stickley and Koyanagi, 2016) and specifically among the elderly (Wiktorsson et al., 2010). Since marriage was found to be a protective factor, whereas being divorced, widowed, or single increased the risk for depression, mental pain, and suicide (Erlangsen et al., 2015; Zhang and Li, 2011), our focus is on the effect of social factors as mediators in the association between pain and suicidality in two separate groups (i.e. married and single).

In this study, we examined the following hypotheses:

1. Physical pain will be associated with suicide ideation; higher levels of physical pain will be associated with higher levels of suicidal ideation.

2. Physical pain will be associated with loneliness and social integration; higher levels of physical pain will be associated with higher levels of loneliness and lower levels of social integration.

3. Social integration and loneliness will be related to suicidal ideation. Higher levels of suicidal ideation will be positively associated with loneliness and negatively with social integration.
4. Social integration and loneliness will mediate the association between physical pain and suicidal ideation.

5. Marital status will moderate the mediation of social integration and loneliness in the association between physical pain and suicidal ideation. The mediation model will be significant among single, but not married, elderly men.

\section{Methods}

\section{Participants and procedure}

The present study is part of a larger research project, which proposes to investigate a variety of risk and protective factors for suicide in the elderly. Participants were 198 elderly men in Israel (age 65-94; $M=76.14$, standard deviation, $\mathrm{SD}=8.05)$. Most of them were married (68\%), with some widowed (20\%), divorced $(8 \%)$, in a relationship $(3 \%)$, and single $(1 \%)$. Most of the participants were living in their house $(72 \%)$, while the rest were in assisted living residences and nursing homes $(28 \%)$. About half of our sample were born in Israel $(47 \%)$. Similar to previous studies (e.g. Heisel and Flett, 2016), participants were recruited through advertisements on social network sites, official requests to managers of organizations for the elderly, and snowball method, in which the recruited participants refer to potential others. This sampling method is considered to be appropriate for studying hard-toreach populations (Biernacki and Waldorf, 1981; Heckathorn, 2011). Inclusion criteria were at least 8 years of education $(M=14.14, S D=3.68)$, Hebrew at the level of mother tongue, and no significant cognitive impairment (Mini Mental State Examination $[M M S E]>20$ ). Exclusion criteria were severe physical or mental disability and custodian or full-time worker as a caregiver.

The study was approved by the institutional review board of Ariel University. A procedure was designed to include actions to be taken in case of suicide risk. Each potential participant was approached by phone in order to provide an explanation of the study and conduct initial screening. Data collection was conducted at participant homes. First, the MMSE was administered, in order to examine participant cognitive status, as part of the screening procedure. Participants with an intact cognitive state continued to the next phase, in which they were asked to fill out the questionnaires. Finally, participants were debriefed and received information regarding further optional support.

\section{Measures}

Beck Scale for Suicidal Ideation (BSS) (BECK ET AL., 1988)

This is a 21-item self-report questionnaire that assesses current suicidal ideation (items 1-19) and 
past suicidal behavior (items 20-21). We used this measure to assess suicidal ideation; therefore, only the 19 suicidal ideation items were used. Total scores could range from 0 to 48 ; higher scores indicated higher suicidal ideation but there are no specific cutoff scores to classify the severity of suicidal risk (Beck and Steer, 1991). BSS has shown high internal consistency and was found to have strong convergent validity (Beck et al., 1988).

Physical pain was measured by the 3-item subscale from the WHO Study on global AGEing and adult health (SAGE) (2007). Participants were asked to rate the severity of physical pain experienced in the previous month using a 5-point Likert scale. The scale showed high internal consistency (Cronbach's $\alpha=.89$ ).

Multidimensional Social Integration in Later Life Scale (SILlS) (Fuller-Iglesias AND RajBhandaRi, 2016)

The original tool consists of two subscales: frequency and satisfaction of various social integration-related activities. In our study, we used the "frequency" subscale, which consists of 10 items that are rated on a 5-point Likert scale. The scale showed acceptable internal consistency (Cronbach's $\alpha=.75$ ).

\section{The UCla Loneliness Scale (Version 3)}

(Russel, 1996)

The original tool (University of California, Los Angeles (UCLA) Loneliness Scale) consists of 20 items assessing the experience of loneliness on a 4-point Likert scale (Russell et al., 1980). Version 3 is the short version of this scale, and it consists of 10 items. The internal consistency of this scale in the present study was good (Cronbach's $\alpha=.84$ ).

\section{Statistical analysis}

The relationships between physical pain, loneliness, social integration, and suicidal ideation were explored using correlations and PROCESS (Hayes, 2013; Preacher and Hayes, 2008). In the mediation model, the contribution of loneliness and social integration to the understanding of the association between physical pain and suicidal ideation was examined separately. In order to explore whether the relationship between physical pain and suicidal ideation is dependent on marital status, moderated mediation analysis was used. Therefore, the role of loneliness and social integration in the understanding of the association between physical pain and suicidal ideation was examined in two separate groups: married (i.e. being married or in a marital relationship) and single (i.e. single, widowed, or divorced). The moderating effect of marital status on the mediation model (physical pain - loneliness
Table 1. Descriptive statistics

\begin{tabular}{|c|c|c|}
\hline & $\mathrm{N} / \mathrm{M}$ & $\% / \mathrm{SD}$ \\
\hline \multicolumn{3}{|l|}{ BSS } \\
\hline 0 & 116 & $58 \%$ \\
\hline greater than 0 & 84 & $42 \%$ \\
\hline \multicolumn{3}{|l|}{ Marital status } \\
\hline Married or in relationship & 58 & $29 \%$ \\
\hline Single, divorced or widowed & 142 & $71 \%$ \\
\hline \multicolumn{3}{|l|}{ Housing } \\
\hline Independent & 57 & $28.5 \%$ \\
\hline Non-residential & 143 & $71.5 \%$ \\
\hline SES & 2.53 & 0.83 \\
\hline Age & 76.14 & 8.05 \\
\hline Education & 14.14 & 3.68 \\
\hline Pain & 1.89 & 1.03 \\
\hline UCLA & 1.91 & 0.56 \\
\hline SILLS & 3.11 & 0.75 \\
\hline
\end{tabular}

BSS $=$ Beck Scale for Suicidal Ideation; SES $=$ socioeconomic status; UCLA = University of California, Los Angeles; SILLS = Multidimensional Social Integration in Later Life.

and social integration - suicidal ideation) was evaluated using the moderated mediation index presented by Hayes (2015). Confidence interval for the index was generated by PROCESS macro. When the confidence interval does not include zero, the moderated mediation model is considered as significant. In order to estimate the mediation effect, we used the bootstrapping method. All analyses were conducted using SPSS v.24 software.

Using G*Power (Faul et al., 2009) to run power calculations, we determined that for a moderated mediation analysis with four predictors, a sample size of at least 602 participants was needed to predict a small effect size (Cohen's f2) of 0.02 at an $\alpha$ level of 0.05 , with a power of $0.80 ; 85$ participants were needed for detection of medium effect (0.15) and 40 participants for large effect (0.35).

\section{Results}

Demographic characteristics and the descriptive statistics of the study variables are presented in Table 1. Some level of suicidal ideation (BSS $>0$ ) was reported by $42 \%(n=84)$ of the sample. The BSS has no validated cutoff scores that categorize the severity of suicidal risk; increasing scores indicate greater suicide risk, while any positive score reflects significant risk (Beck and Steer, 1991). Since the distribution of suicidal ideation was not normal (Kurtosis $=17.81$, skewness $=3.80$ ), it was transformed to a dichotomous variable: participants with or without some level of suicidal ideation (Miller et al., 2001). Table 2 presents the relationships between study variables. As can be seen, 
Table 2. Correlation matrix

\begin{tabular}{|c|c|c|c|c|c|c|c|c|c|}
\hline & BSS & MARITAL STATUS & HOUSING & SES & AGE & EDUCATION & PAIN & UCLA & SILLS \\
\hline BSS & - & & & & & & & & \\
\hline Marital status & .07 & - & & & & & & & \\
\hline Housing & -.07 & $-.60^{* * *}$ & - & & & & & & \\
\hline SES & .02 & $-.21^{* *}$ & $.22^{* *}$ & - & & & & & \\
\hline Age & -.08 & $-.39^{* * * *}$ & $.52^{* *}$ & .08 & - & & & & \\
\hline Education & .10 & $.23^{* * *}$ & $-.26^{* *}$ & $-.35^{* * *}$ & $-.24^{* * *}$ & - & & & \\
\hline Pain & $.19^{* *}$ & -.11 & .08 & $.17^{*}$ & .06 & -.14 & - & & \\
\hline UCLA & $.20^{* *}$ & -.05 & .08 & .13 & .08 & .05 & $.33^{* * *}$ & - & \\
\hline SILLS & $-.19^{* *}$ & .05 & -.09 & -.06 & -.00 & .03 & $-.27^{* * *}$ & $-.42^{* * *}$ & - \\
\hline
\end{tabular}

${ }^{*} \mathrm{p}<.05,{ }^{* *} \mathrm{p}<.01,{ }^{* * *} \mathrm{p}<.001$. BSS $=$ Beck Scale for Suicidal Ideation. Dichotomous measure was used: $0=0,1=$ every score greater than 0 . Frequency of the last category is presented; marital status: $0=$ married or in relationship, $1=$ single, divorced, or widowed. Frequency of the first category is presented; housing: $0=$ independent, $1=$ non-residential. Frequency of the first category is presented; SES $=$ socioeconomic status; UCLA = University of California, Los Angeles; SILLS = Multidimensional Social Integration in Later Life.

Table 3. Mediation models for the relationship between pain and suicidal ideation

\begin{tabular}{|c|c|c|c|c|c|c|c|c|c|}
\hline \multirow[b]{2}{*}{ GROUP } & \multirow[b]{2}{*}{ MEDIATOR } & \multicolumn{2}{|c|}{ РATH A } & \multicolumn{2}{|c|}{ РАТН В } & \multicolumn{2}{|c|}{ РАTH C } & \multicolumn{2}{|c|}{$\begin{array}{l}\text { INDIRECT EFFECT } \\
\text { (BOOTSTRAPPING) }\end{array}$} \\
\hline & & B (SE) & $\mathrm{T}$ & $\mathrm{B}(\mathrm{SE})$ & z & $\mathrm{B}(\mathrm{SE})$ & $\mathrm{z}$ & в $(S E)$ & $95 \% \mathrm{CI}$ \\
\hline \multicolumn{10}{|c|}{ All samples $(n=198)$} \\
\hline & UCLA & $.18(.04)$ & $4.95^{* * *}$ & $.60(.29)$ & $2.09^{*}$ & $.29(.15)$ & 1.91 & $.11(.06)$ & $.01 ; .23$ \\
\hline & SILLS & $-.19(.05)$ & $-3.90^{* * *}$ & $-.42(.21)$ & $-2.01^{*}$ & $.31(.15)$ & $2.08^{*}$ & $.08(.05)$ & $.00 ; .22$ \\
\hline \multicolumn{10}{|c|}{ Single, divorced, and widowed $(n=58)$} \\
\hline & UCLA & $.17(.07)$ & $2.45^{*}$ & $1.24(.54)$ & $2.29^{*}$ & $.25(.27)$ & 0.94 & $.21(.12)$ & $.03 ; .50$ \\
\hline & SILLS & $-.21(.09)$ & $-2.45^{*}$ & $-.98(.43)$ & $-2.26^{*}$ & $.23(.26)$ & 0.90 & $.21(.17)$ & $.01 ; .67$ \\
\hline \multicolumn{10}{|c|}{ Married and in relationship $(n=140)$} \\
\hline & UCLA & $.18(.04)$ & $4.26^{* * *}$ & $.31(.36)$ & .86 & $.36(.19)$ & 1.85 & $.06(.07)$ & $-.08 ; .22$ \\
\hline & SILLS & $-.18(.06)$ & $-2.91^{* *}$ & $-.22(.25)$ & -.90 & $.38(.19)$ & $1.97^{*}$ & $.04(.05)$ & $-.02 ; .19$ \\
\hline
\end{tabular}

${ }^{*} \mathrm{p}<.05,{ }^{* *} \mathrm{p}<.01,{ }^{* * *} \mathrm{p}<.001$. Path $\mathrm{a}=$ direct effect of pain on the mediator; Path $\mathrm{b}=$ direct effect of the mediator on suicidal ideation; Path $\mathrm{c}=$ direct effect of pain on suicidal ideation. The coefficients are unstandardized. UCLA = University of California, Los Angeles; SILLS $=$ Multidimensional Social Integration in Later Life.

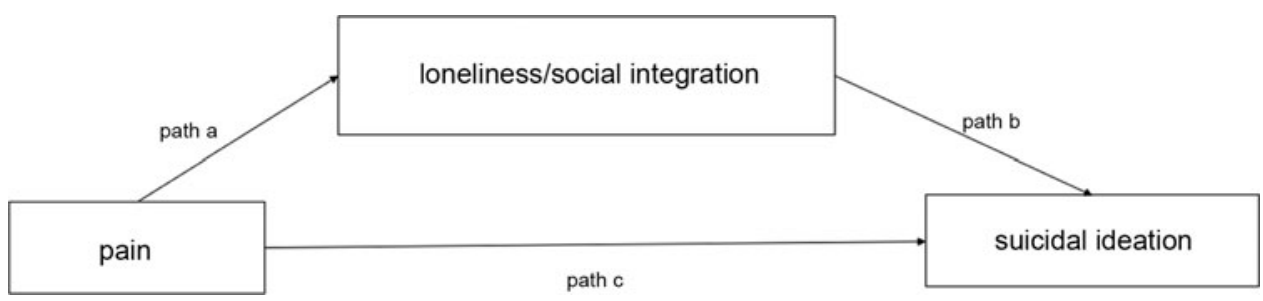

Figure 1. Moderated mediation proposed model: Loneliness and social integration as mediators in the association between pain and suicidal ideation.

physical pain, loneliness, and social integration were significantly related to suicidal ideation.

The mediation effect of loneliness and of social integration on the relationship between physical pain and suicidal ideation was explored separately, due to the relatively high correlation between the UCLA and SILLS scores (see Figure 1 for the proposed models). Basic mediation models were tested in the first stage, and moderated mediation models, taking into account marital status, were tested second.

The unstandardized path coefficients indicating the direct effects of pain on the mediators (i.e. loneliness and social integration), the direct effects of the mediators on suicidal ideation, and the direct effect of pain on suicidal ideation are presented in Table 3. The estimations of the indirect effects, including the bootstrapping confidence intervals 
(CIs), are also presented. As can be seen in the two upper rows of the table, both loneliness and social integration significantly mediated the relationship between physical pain and suicidal ideation in the total sample.

Simple mediation models for each marital status group are presented in the lower rows of Table 3. Significant moderated mediation was not found in the UCLA model (moderated mediation index = -0.16 , standard error, $\mathrm{SE}=0.13$, bootstrapping $95 \% \mathrm{CI}=-0.45,0.08)$, nor in the SILLS model (moderated mediation index $=-0.14, \mathrm{SE}=0.11$, bootstrapping $95 \% \mathrm{CI}=-0.41,0.01)$. However, as can be seen in Table 3, loneliness and social integration significantly mediated the relationship between physical pain and suicidal ideation among single, divorced, and widowed participants but not among participants in a relationship or married. The relationship between loneliness and social integration and suicidal ideation was significant only in the first group, not in the second.

\section{Discussion}

The aim of our study was to examine the role of loneliness and social integration as mediators in the association between physical pain and suicidal ideation among single and married elderly men. Loneliness and social integration were found to significantly mediate the relationship between physical pain and suicidal ideation. The magnitude of the effects was small but of potential theoretical interest. These findings suggest that elderly men who suffer from physical pain may be less socially integrated, feel lonelier, and are, therefore, more prone to experience suicidal ideation, especially if they are single.

Previous research consistently pointed at physical health difficulties as well as at loneliness and poor social integration as risk factors for suicide (Erlangsen et al., 2015; Waern et al., 2003). Our results suggest a possible bio-psycho-social explanation by which physical pain contributes to the experience of both objective and subjective social isolation, and these in turn may lead to suicidal thoughts among elderly single men. It is important to note that this mediation model was found to be significant in the single group only. Being single is a well-known risk factor of suicide in all ages (Corcoran and Nagar, 2010). However, single men may be facing a particular set of age-related challenges. In late life, absence of a spouse representing a significant interpersonal loss may lead to continuous grief and emptiness (Ryckebosch-Dayez et al., 2016). In addition, absence of a spouse may be associated with unfulfilled interpersonal needs and hence lead to greater levels of emotional distress, heightened feelings of loneliness, and lower levels of social integration (Stessman et al., 2014; Berkman et al., 2000). Moreover, it is possible that physical pain in elderly men who are single may be experienced as more emotionally distressful. In sum, among elderly single men there may be a gap between the resources needed for dealing with physical pain and the extent of available interpersonal support, and this gap may elevate their risk of suicide.

The findings in this study have several practical and clinical implications. Our main conclusion is that single elderly men who suffer from physical pain are at higher suicide risk due to a potential increased experience of loneliness and decreased social integration, among other factors. This conclusion, if replicated in other studies, can serve as a basis for raising awareness among the public as well as among professionals, about the relationship of physical pain with suicidal risk, due to its association with reduced social activity and increased feelings of loneliness, and about the need to address the social needs of single elderly suffering from physical pain. Such awareness may allow for more integrative care for the elderly with physical problems, one that also addresses psychosocial factors. In cases of ongoing physical pain, it is advisable to incorporate specific medical or psychological interventions designed to allow better pain management. Welfare systems should create and implement special and accessible community intervention programs, in order to reduce social isolation among elderly men coping with physical pain. In some cases, psychotherapy is likely to be needed to enable the elderly to improve their relationship with their family and friends as well as increase their participation in social activities and their social support.

This study has several limitations. First, it is possible that suicidality was underreported in our sample, due to the known tendency of denial of emotional stress and suicidal behavior in this population (Van Orden et al., 2012). Another limitation is that recruitment was based on voluntary consent in the community; it is possible that participants in our study were more disposed to share their emotional experiences than the general elderly population. In addition, the cross-sectional nature of the study does not allow inferences about causal relationships between variables. Finally, it is important to note that although we examined a model in which pain contributes to increased loneliness and this in turn contributes to suicide ideation, correlations are bidirectional, so conclusions about directionality cannot be definitively made. In other words, it is possible that depression and suicidal ideation contribute to isolation, and hence to higher levels of loneliness and even to heightened physical 
pain. Indeed, elderly men who commit suicide may keep distance from the society and relatives (Kjølseth et al., 2009). In addition, loneliness is associated with adverse health consequences, fatigue, and pain (Luanaigh and Lawlor, 2008; Jaremka et al., 2013).

Following these considerations, future studies should further examine the role of physical pain as well as psychological and social factors on the social integration and loneliness of elders. Our hope is that the present study will contribute to the extension of a bio-psycho-social approach to a relatively neglected area, i.e. suicidality in elderly people.

\section{Conflict of interest}

None declared.

\section{Description of authors' roles}

Ms. Lutzman designed the study, collected the data, was responsible for statistical analysis, and wrote the manuscript. Dr. Sommerfeld supervised the study and wrote the manuscript. Prof. Ben-David supervised the process and assisted with writing the manuscript.

\section{Acknowledgments}

We would like to thank Ariel University for supporting this work.

\section{References}

Bahk, W.M., Park, S., Jon, D.I., Yoon, B.H., Min, K.J. and Hong, J.P. (2011). Relationship between painful physical symptoms and severity of depressive symptomatology and suicidality. Psychiatry Research, 189, 357-361.

Beck, A.T. and Steer, R.A. (1991). Manual for Beck Scale for Suicidal Ideation. New York: Psychological Corporation.

Beck, A.T., Steer, R.A. and Ranieri, W.F. (1988). Scale for suicidal ideation: psychometric properties versus respondent-driven sampling. Sociological Methodology, $41,355-366$.

Biernacki, P. and Waldorf, D. (1981). Snowball sampling: problems and techniques of chain referral sampling. Sociological Methods E Research, 10, 141-163.

Domènech-Abella, J., Mundó, J., Haro, J.M., and Rubio-Valera, M. (2019). Anxiety, depression, loneliness and social network in the elderly: longitudinal associations from The Irish Longitudinal Study on Ageing (TILDA). Fournal of Affective Disorders, 246, 82-88.

Hayes, A.F. (2015). An index and test of linear moderated mediation. Multivariate Behavioral Research, 50, 1-22.

Heckathorn, D.D. (2011). Comment: snowball versus respondent-driven sampling. Sociological Methodology, 41, 355-366.

Heisel, M.J. and Flett, G.L. (2016). Does recognition of meaning in life confer resiliency to suicide ideation among community-residing older adults? A longitudinal investigation. The American fournal of Geriatric Psychiatry, 24, 455-466.

Jaremka, L.M., Andridge, R.R., Fagundes, C.P., et al. (2013). Pain, depression, and fatigue: loneliness as a longitudinal risk factor. Health Psychology, 19, 19.

Kim, S.H. (2016). Suicidal ideation and suicide attempts in older adults: influences of chronic illness, functional limitations, and pain. Geriatric Nursing, 37, 9-12.

Kjølseth, I., Ekeberg, O. and Steihaug, S. (2009). "Why do they become vulnerable when faced with the challenges of old age?" Elderly people who committed suicide, described by those who knew them. International Psychogeriatrics, 21, 903-912.

Law, C. K, Kolves, K. and De Leo, D. (2016). Influences of population-level factors on suicides in older adults: a national ecological study from Australia. International fournal of Geriatric Psychiatry, 31, 384-91.

Lee, J.Y. and Moon, Y.S. (2015). Effects of chronic pain and social support on depression and suicide in the elderly. Fournal of Digital Convergence, 13, 445-458.

Lewitzka, U., Spirling, S., Ritter, D., et al. (2017). Suicidal ideation vs. suicide attempts: clinical and psychosocial profile differences among depressed patients: a study on personality traits, psychoapthological variables, and sociodemograpic factors in 228 patients. Fournal of Nervous and Mental Disorders, 205(5), 361-371.

Luanaigh, C. and Lawlor, B. (2008). Loneliness and the health of older people. International fournal of Geriatric Psychiatry, 23, 1213-1221.

Miller, J.S., Segal, D.L. and Coolidge, F.L. (2001). A comparison of suicidal thinking and reasons for living among younger and older adults. Death Studies, 25, 357-365.

Preacher, K.J. and Hayes, A.F. (2008). Asymptotic and resampling strategies for assessing and comparing indirect effects in multiple mediator models. Behavior Research Methods, 40(3), 879-891.

Racine, M. (2018). Chronic pain and suicide risk: a comprehensive review. Progress in Neuro-psychopharmacology and Biological Psychiatry, 87, 269-280.

Rapo-Pylkko, S., Haanpaa, M. and Liira, H. (2016). Chronic pain among community-dwelling elderly: a population-based clinical study. Scandinavian fournal of Primary Health Care, 34, 159-164.

Russell, D., Peplau, L.A. and Cutrona, C. (1980). The revised UCLA loneliness scale: concurrent and discriminant validity evidence. Fournal of Personality and Social Psychology, 39, 472-480. 
Russel, D.W. (1996). UCLA loneliness scale (Version 3): reliability, validity, and factor structure. Fournal of Personality Assessment, 66, 20-40.

Ryckebosch-Dayez, A., Zech, E., Mac Cord, J. and Taverne, C. (2016). Daily life stressors and coping strategies during widowhood: a diary study after one year of bereavement. Death Studies, 40, 461-478. doi: 10.1080/ 07481187.2016.1177750.

Stessman, J., Rottenberg, Y., Shimshilashvili, I., Ein-Mor, E. and Jacobs, J.M. (2014). Loneliness, health, and longevity. The fournals of Gerontology Series A: Biological Sciences and Medical Sciences, 69, 744-750.

Stickley, A. and Koyanagi, A. (2016). Loneliness, common mental disorders and suicidal behavior: findings from a general population survey. Fournal of Affective Disorders, 197, 81-87.

Theeke, L.A. (2009). Predictors of loneliness in U.S. adults over age sixty-five. Archives of Psychiatric Nursing, 23, 387-396.

Van Orden, K.A., Bamonti, P.M., King, D.A. and Duberstein, P.R. (2012). Does perceived burdensomeness erode meaning in life among older adults? Aging and Mental Health, 16, 855-860.

Waern, M., Rubenowitz, E., Runeson, B., Skoog, I., Wilhelmson, K. and Allebeck, P. (2002). Burden of illness and suicide in elderly people: case-control study. British Medical fournal, 324, 1-4.

Waern, M., Rubenowitz, E. and Wilhelmson, K. (2003). Predictors of suicide in the old elderly. Gerontology, 49, 328-334.

Wiktorsson, S., Runeson, B., Skoog, I., Ostling, S. and Waern, M. (2010). Attempted suicide in the elderly: characteristics of suicide attempters 70 years and older and a general population comparison group. American fournal of Geriatric Psychiatry, 18, 57-67.

World Health Organization. (2007). WHO Study on global AGEing and adult health (SAGE). Available at: https://www .who.int/healthinfo/sage/indepth/en/; last accessed 23 June 2020.

World Health Organization. (2014). Preventing suicide: A global imperative. Available at: http://apps.who.int/iris/ bitstream/10665/131056/1/9789241564779_eng.pdf? ua $=1$; last accessed 23 June 2020.

Zhang, B. and Li, J. (2011). Gender and marital status differences in depressive symptoms among elderly adults: the roles of family support and friend support. Aging $\mathcal{E}$ Mental Health, 15(7), 844-854.

Zhang, Z. and Zhang, J. (2015). Social participation and subjective well-being among retirees in China. Social Indicators Research, 123(1), 143-160. 